\title{
Transmission of Antimicrobial- Resistant Staphylococcus aureus Clonal Complex 9 between Pigs and Humans, United States
}

Pranay R. Randad, Jesper Larsen, Hülya Kaya, Nora Pisanic, Carly Ordak, Lance B. Price, Maliha Aziz, Maya L. Nadimpalli, Sarah Rhodes, Jill R. Stewart, Dave C. Love, David Mohr, Meghan F. Davis, Lloyd S. Miller, Devon Hall, Karen C. Carroll, Trish M. Perl, Christopher D. Heaney

Transmission of livestock-associated Staphylococcus aureus clonal complex 9 (LA-SA CC9) between pigs raised on industrial hog operations (IHO) and humans in the United States is poorly understood. We analyzed wholegenome sequences from 32 international $S$. aureus CC9 isolates and 49 LA-SA CC9 isolates from IHO pigs and humans who work on or live near IHOs in 10 pig-producing counties in North Carolina, USA. Bioinformatic analysis of sequence data from the 81 isolates demonstrated 3 major LA-SA CC9 clades. North Carolina isolates all fell within a single clade (C3). High-resolution phylogenetic analysis of $\mathrm{C} 3$ revealed 2 subclades of intermingled $\mathrm{IHO}$ pig and human isolates differing by $0-34$ single-nucleotide polymorphisms. Our findings suggest that LA-SA CC9 from pigs and humans share a common source and provide evidence of transmission of antimicrobial-resistant LA-SA $\mathrm{CC} 9$ between IHO pigs and humans who work on or live near IHOs in North Carolina.

L ivestock-associated Staphylococcus aureus (LA-SA) has emerged among pigs raised in industrial hog operations (IHOs) and persons who work on or live near IHOs globally, including in the United States (1-4). IHO workers who are occupationally exposed

Author affiliations: Johns Hopkins University, Baltimore, Maryland, USA (P.R. Randad, N. Pisanic, C. Ordak, D.C. Love, D. Mohr,

M.F. Davis, L.S. Miller, K.C. Carroll, T.M. Perl, C.D. Heaney);

Statens Serum Institut, Copenhagen, Denmark (J. Larsen,

H. Kaya); George Washington University, Washington, DC, USA

(L.B. Price, M. Aziz); Tufts University, Boston, Massachusetts,

USA (M.L. Nadimpalli); University of North Carolina at Chapel

Hill, Chapel Hill, North Carolina, USA (S. Rhodes, J.R. Stewart);

Rural Empowerment Association for Community Help (REACH),

Warsaw, North Carolina, USA (D. Hall); University of Texas

Southwestern Medical Center, Dallas, Texas, USA (T.M. Perl)

DOI: https://doi.org/10.3201/eid2703.191775 to pigs are at increased risk for intranasal carriage of $S$. aureus, including methicillin-resistant $S$. aureus (MRSA), multidrug-resistant S. aureus (MDRSA), and LA-SA $(3,5)$. Furthermore, persons exposed to LA-SA are at risk of developing mild-to-severe infections, including skin and soft tissue infections (SSTIs), pneumonia, endocarditis, osteomyelitis, and bacteremia (5-8). Recent evidence supports emergence of diverse clones associated with IHOs. S. aureus clonal complex 9 (CC9), for example, has been reported as a dominant LA-SA lineage in Asia and has been described as an emerging clone in some areas with intensive industrial livestock production in the United States (9-11).

The population structure and transmission dynamics of emerging LA-SA CC9 strains in the United States remains poorly understood. Previous epidemiologic studies in the top 10 pig-producing counties in North Carolina, the second leading US pig-producing state, showed a high prevalence of LA-SA CC9 nasal carriage among IHO pigs and IHO workers $(3,12)$. Epidemiologic findings provide support for potential transmission of LA-SA CC9 between IHO workers and their household contacts, including minor children ( $<18$ years of age; IHO minors), based on nasal carriage of LA-SA CC9 with concordant spa types at the same time point (3). Epidemiologic studies have also identified instances of LA-SA CC9 nasal carriage among community residents with no known exposure to livestock in high-density IHO areas of North Carolina (2). Whole-genome sequencing (WGS) analysis provides an opportunity to characterize the population structure and transmission dynamics of LA-SA CC9 in the United States. The objectives of this study were to use WGS and phylogenetic analyses to elucidate the population structure of S. aureus CC9 from various regions in North America, South America, 
Europe, and Asia and to investigate potential transmission of antimicrobial-resistant LA-SA CC9 among IHO pigs and humans who work on or live near IHOs in North Carolina.

\section{Methods}

\section{Sources of S. aureus Isolates from Humans and from Pigs Raised on IHOs in North Carolina}

$S$. aureus isolates from IHO pigs were collected from a convenience sample of a single IHO in North Carolina (IHO-1), as described previously (12). We collected additional pig samples by hanging a length of undyed, unbleached cotton rope in pig pens of $20 \mathrm{IHOs}$ in North Carolina (IHO-2-IHO-21) (Appendix). Pig isolates were recovered from IHO-2, IHO-3, IHO-4, IHO-5, and IHO-6 for a total of 6 IHOs (IHO-1-IHO6). Isolates from IHO-2-IHO-6 have not been published previously. The spa type for all IHO pig isolates was characterized, as previously described (12), and used to assign each isolate to a putative multilocus sequence type (MLST).

$S$. aureus isolates from humans were collected from participants who were previously enrolled into 1 of 3 separate epidemiologic studies (study 1, study 2 , and study 3 ) and screened for nasal carriage of $S$. aureus (Appendix, https://wwwnc.cdc.gov/EID/ article/27/3/19-1775-App1.pdf). Sample collection, sample processing, and $S$. aureus isolation methods were described previously (1-3). MLST was previously determined for all study 1 isolates (1). The spa type was previously characterized for study 2 and study 3 isolates and used to assign a putative MLST based on previously published associations between spa types and MLSTs $(2,3)$.

\section{Selection of S. aureus CC9 Isolates for WGS Analysis}

A total of 236 putative or MLST-confirmed S. aureus CC9 isolates were recovered from IHO pigs $(n=91)$ and humans $(\mathrm{n}=145)$ in North Carolina during 20112016 (Appendix). For this study, a convenience sample of 49 isolates from North Carolina were subjected to WGS analysis, including 10 isolates from pigs raised on 4 different IHOs, 34 isolates from $25 \mathrm{IHO}$ workers, 1 isolate each from 3 IHO minors, and 1 isolate each from 2 community resident adults (Appendix). For comparative purposes, we also included an international collection of $32 \mathrm{~S}$. aureus CC9 genomes available as of August 1, 2018, from the National Center for Biotechnology Information (NCBI) Reference Sequence Database (http://www.ncbi.nlm.nih.gov/ RefSeq), which included information on source, geographic location, and collection year.

\section{WGS and Bioinformatic Analyses}

We prepared DNA for multiplexed, paired-end sequencing by preparing libraries using either the Nextera XT DNA Library Preparation Kit (Illumina, Inc.), according to manufacturer instructions, or the Kapa Hyper Prep Kit (Kapa Biosystems, Inc., https:/ / www.sigmaaldrich.com) and uniquely barcoded adaptors from NEXTFLEX-96 Unique Dual Index barcodes (Bioo Scientific, https://www.biooscientific.com). We prepared equimolar pools of $S$. aureus libraries at a concentration of $2 \mathrm{nmol}$ and sequenced on a MiSeq (Illumina, Inc., https:/ / ww.illumina.com) at $2 \times 300 \mathrm{bp}$. WGS data are available in the NCBI Sequence Read Archive (http://www.ncbi.nlm.nih. gov; BioProject no. PRJNA574434).

We used SPAdes (13) to generate de novo assemblies and compared these against the $S$. aureus MLST database (14) to assign MLSTs. We used ABRicate (https://github.com/tseemann/abricate) to search the ResFinder database for antimicrobial-resistance (AMR) genes (15). We used BLASTN (https://blast.ncbi.nlm.nih.gov/Blast.cgi) to detect genes in the phage-associated immune evasion cluster (IEC), including scn, chp, sak, sea (GenBank accession no. NC_009641), and sep (GenBank accession no. BA000018) (16).

We used the NASP pipeline (17) to map sequence reads against the de novo-assembled genome of North Carolina isolate IHOW6.1 (BioProject accession no. PRJNA574434) and to perform single-nucleotide polymorphism (SNP) calling, as described previously (8). We used Gubbins version 2.3.1 (18) to remove recombination from the SNP alignment and used the remaining SNPs in the core genome to construct a midpointrooted maximum-likelihood tree by using PhyML (19) with a general time-reversible model of nucleotide substitution and 100 bootstrap replicates (20). We used the same methods to perform a separate SNP analysis of the cluster containing the North Carolina isolates (clade 3) to improve the resolution of the transmission analysis. We calculated pairwise SNP differences by using MEGA5 (21). To define a SNP-based threshold for assigning isolates into putative transmission clusters, we used the maximum within-farm pairwise SNP distance among $S$. aureus CC9 isolates from IHO-1, in which all isolates were collected from the same IHO at the same sampling time.

\section{Antimicrobial Susceptibility Testing}

Isolates in the North Carolina collection previously were assessed for susceptibility to a panel of antimicrobial drugs by using the Phoenix Automated Microbiology System (Becton Dickinson, https://www. 
bd.com) or the Kirby-Bauer disk diffusion method (Appendix Table 2). Testing was completed by the Clinical Microbiology Laboratory at the Johns Hopkins Hospital based on Clinical Laboratory Standards Institute (CLSI; https://clsi.org) guidelines specified in the source studies (1-3) (Appendix Table 2). We defined MDRSA as $S$. aureus isolates resistant to $\geq 3$ classes of antimicrobial drugs (22). We defined MRSA as $S$. aureus harboring the mecA gene.

\section{Statistical analysis}

We used the $\chi^{2}$ test to compare AMR and IEC genes between groups. We performed all statistical analyses by using Stata version 14.2 (StataCorp LLC, https:/ / www.stata.com).

\section{Results}

All 49 isolates from the North Carolina collection used in WGS analysis were classified as sequence type 9 by MLST. Among 81 S. aureus CC9 isolates analyzed, 95\% (77/81) were located in 3 major clades, C1, C2, and C3 (Figure 1; Appendix Tables 3, 4). Despite the small number of pig isolates, each clade contained both pig and human isolates (Figure 1; Appendix Table 3).

Among C1-C3 isolates, 61\% (47/77) contained tetracycline resistance genes. By contrast, only 1 $(1.3 \%)$ of the isolates in C1-C3 contained IEC genes (Figure 1; Appendix Table 4). The presence of pig isolates coupled with the absence of IEC genes and presence of tetracycline resistance genes in $\mathrm{C} 1-\mathrm{C} 3$ suggest that $\mathrm{C} 1-\mathrm{C} 3$ isolates may be members of a larger LA-SA CC9 clade. LA-MRSA CC9, which harbored the mecA gene, was present in $\mathrm{C} 1$ and $\mathrm{C} 2$ but absent from C3.

C1 was composed of isolates primarily originating from Asia (12/13 isolates; $92 \%$ ), of which $46 \%$ $(6 / 13)$ were from China and $46 \%(6 / 13)$ were from Taiwan (Figure 1; Appendix Table 3). All of C2 (14/14 isolates) was composed of isolates originating from Europe, of which $71 \%(10 / 14)$ were from Germany, $21 \%(3 / 14)$ were from the Netherlands, and 7\% (1/14) were from Denmark (Figure 1; Appendix Table 3). C3 included $100 \%(49 / 49)$ of the North Carolina isolates, which made up 98\% (49/50) of all C3 isolates (Figure 1; Appendix Table 3). Only 2 isolates grouped into a clade that did not correspond to the continent of predominance within the clade. A single isolate from Colombia (South America) grouped into C3 with isolates from North Carolina, and a single isolate from the Netherlands grouped into $\mathrm{C} 1$ with isolates from Asia (Figure 1; Appendix Table 3).

High-resolution phylogenetic analysis of C3 revealed multiple distinct subclades, one of which contained all 6 IHO-1 pig isolates from North Carolina (Figure 2). The pairwise SNP distance among IHO pig isolates from IHO-1 ranged from 0-43 SNPs. Thus, we used 43 SNPs as the threshold to identify putative transmission clusters and found that 19 isolates fell into 2 distinct putative transmission clusters (Figure 2). The minimum pairwise SNP distance between IHO pig and human isolates within putative transmission clusters ranged from 12-34 SNPs.

Almost all (94.7\%) transmission cluster isolates were classified as MDRSA (Figure 2). Among 19 putative transmission cluster isolates, 14 were recovered from IHO workers, all of which were classified as MDRSA. Among IHO worker isolates, 2 differed from an IHO pig isolate by only 12 SNPs. An IHO worker isolate that was associated with a recent SSTI differed from an IHO pig isolate by only $20 \mathrm{SNPs}$ (Figure 2 ). One transmission cluster isolate was from an adult community resident with no known exposure to livestock; this isolate also was classified as MDRSA (Figure 2). The minimum SNP distance between this isolate and the closest IHO pig isolate was 25 SNPs and it was 22 SNPs from the closest IHO worker isolate. Among 3 isolates from minors, 2 were identical (0 SNP differences) to an isolate from an IHO worker in the same household (Figure 2); 1 of the isolates from a minor was collected at the same sampling time as the IHO worker isolate. Among C3 isolates, we noted genetic determinants conferring resistance to tetracyclines, including tet $(\mathrm{K})$, tet $(\mathrm{L})$, tet $(\mathrm{T})$; macrolides, including $\operatorname{erm}(\mathrm{A}), \operatorname{erm}(\mathrm{C})$; lincosamides, including $\ln u(\mathrm{~A})$; aminoglycosides, including aac6'-aph2", spc, and aadD; and streptogramins, including $v g a(\mathrm{~A})_{\mathrm{LC}}$ (Figure 2 ).

We noted abundant genetic determinants conferring resistance to several antimicrobial classes among C3 isolates, including tetracyclines in 50\% (25/50), macrolides in 56\% (28/50), and aminoglycosides in $62 \%(31 / 50)$ of C3 isolates (Figure 2; Appendix Table 4). Among LA-SA CC9 clades, $50 \%(25 / 50)$ of C3 isolates were uniquely enriched for erm(A) genes, 16\% $(8 / 50)$ for $v g a(\mathrm{~A})_{\mathrm{LC}^{\prime}}, 42 \%(21 / 50)$ for $\ln u(\mathrm{~A})$, and $54 \%$ $(27 / 50)$ for spc (Figure 1; Appendix Table 4). The mecA gene was absent from $\mathrm{C} 3$ but common among $\mathrm{C} 1$ and $\mathrm{C} 2$ isolates.

\section{Discussion}

Our WGS analysis suggests that the clonal expansion of LA-SA CC9 in North Carolina is distinct from that in Asia and Europe and that LA-SA CC9 from IHO pigs and humans in high-density pig-producing counties of North Carolina come from a common pool. Considering the high degree of phylogenetic relatedness among intermingled IHO pig and human 


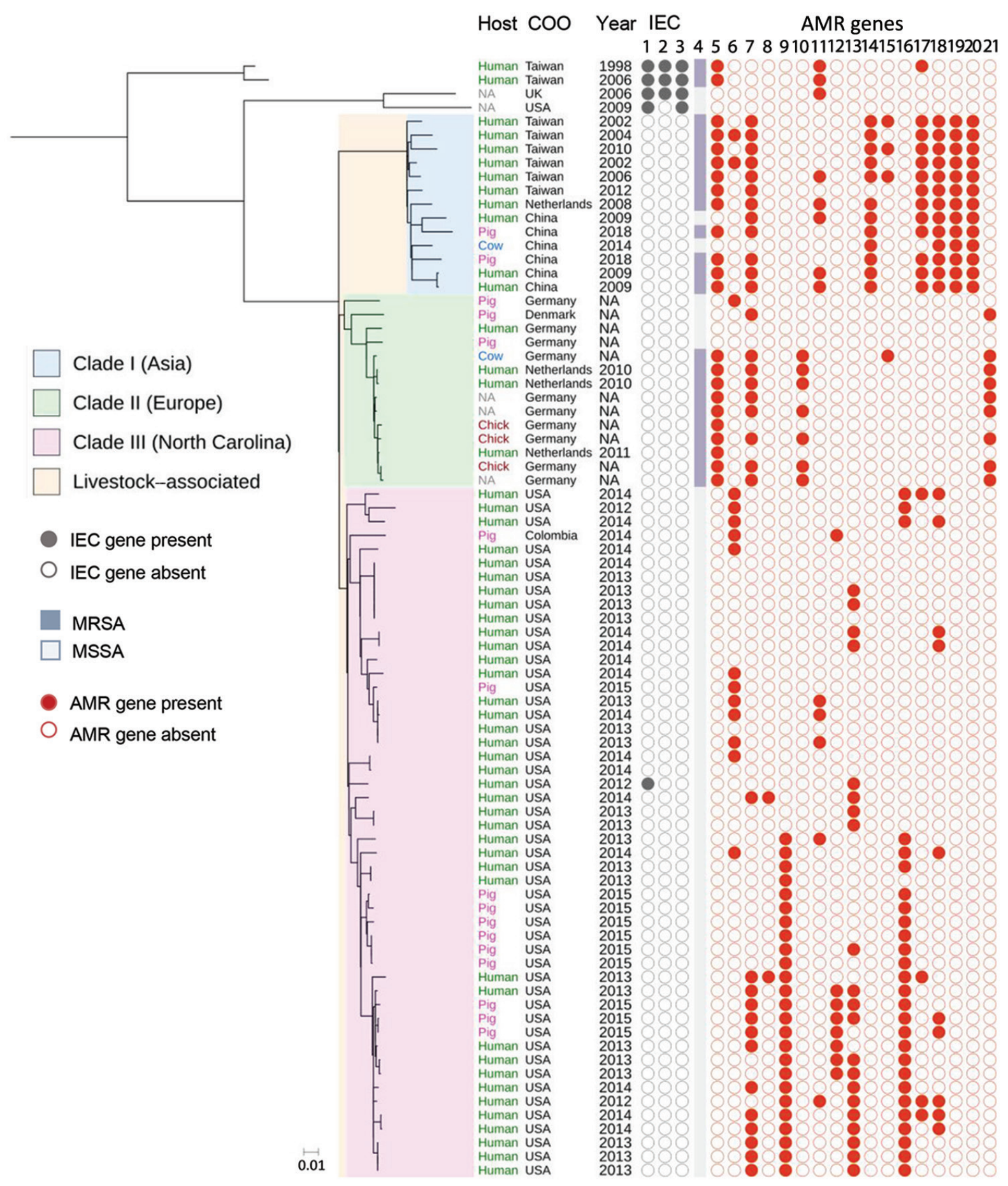

Figure 1. Maximum-likelihood tree demonstrating population structure of Staphylococcus aureus clonal complex (CC) 9 isolates from humans and livestock in North Carolina, USA, and reference sequences. A total of $81 \mathrm{~S}$. aureus CC9 isolates from human and livestock specimens were included in this midpoint-rooted maximum-likelihood phylogeny based on 3,847 core genome single-nucleotide polymorphisms. S. aureus isolates belonged to 3 phylogeographically distinct clades (C1-C3). All the North Carolina collection isolates were included in C3. IEC genes are shown in columns 1, scn; 2, sak; and 3, chp. MRSA is shown in column 4. AMR genes are shown in columns 5, mecA; 6, tet(K); 7, tet(L); 8, tet(T); 9, erm(A); 10, erm(B); 11, erm(C); 12, vga(A) $)_{\mathrm{LC}} ; 13, \ln u(\mathrm{~A}) ; 14, \ln u(\mathrm{~B}) ; 15, \operatorname{str} ; 16, \operatorname{spc}$; 17, aadD; 18, aac(6); 19, ant(6)-1a; 20, dfrG; and 21, dfrK. Scale bar indicates nucleotide substitutions per site. AMR, antimicrobial resistance; Chick, chicken; COO, country of origin; IEC, immune evasion cluster; MRSA, methicillin-resistant S. aureus; MSSA, methicillin-susceptible S. aureus; NA, not applicable. 
isolates in putative transmission clusters, the results of this study support potential transmission of antimicrobial-resistant LA-SA CC9 between IHO pigs and humans in the United States.

Our results also provide evidence of householdlevel transmission of LA-SA CC9 between IHO workers and minors and suggest that potential LA-SA CC9 transmission is not limited to the occupational setting. Dissemination of LA-SA CC9 into the general human population represents a public health concern for 2 reasons. Globally, communities include a higher proportion of children, the elderly, and probably immunocompromised persons, who are at higher risk of developing invasive staphylococcal infections, compared with IHO workers who are predominantly healthy adults of working age. Our analysis revealed an 11-year-old child and an IHO worker residing in the same household who were carrying identical



Figure 2. High-resolution population structure of clade 3 livestock-associated Staphylococcus aureus clonal complex (CC) 9 isolates from humans and livestock in North Carolina, USA, and reference isolates. A subset of 50 livestock-associated S. aureus CC9 isolates that were collected from IHO pigs, IHO workers, IHO minors, and CR adults were included in this midpoint-rooted maximum-likelihood phylogeny based on 1,198 core genome single-nucleotide polymorphisms. A single subclade, denoted as the IHO pig cluster, included only pig isolates from IHO-1 and was used to set a threshold of 43 SNPs for identifying transmission clusters; clusters of IHO pig and human isolates separated by $\leq 43$ SNPs are considered transmission clusters. Two subclades included intermingled human and IHO pig isolates with a high degree of phylogenetic relatedness and were considered transmission clusters. IEC isolates are shown in columns 1 , scn, 2 , sak, and 3, chp. AMR genes are shown in columns 4, tet( $\mathrm{K}) ; 5, \operatorname{tet}(\mathrm{L}) ; 6, \operatorname{tet}(\mathrm{T}) ; 7, \operatorname{erm}(\mathrm{A}) ; 8, \operatorname{erm}(\mathrm{C}) ; 9, \operatorname{vga}(\mathrm{A}) \operatorname{Lc} ; 10, \operatorname{lnu}(\mathrm{A}) ; 11, \operatorname{spc} ; 12$, aadD; and 13, aac(6). MDRSA is shown in column 14. Antimicrobial drug resistance is shown in columns 15 , fluoroquinolone resistance, considered phenotypic resistance to moxifloxacin; 16, lincosamide resistance, considered phenotypic resistance to clindamycin; 17, macrolide resistance, considered phenotypic resistance to erythromycin; 18, aminoglycoside resistance, considered phenotypic resistance to gentamicin; 19, tetracycline resistance, considered phenotypic resistance to tetracycline; and 20, penicillin resistance. Scale bar indicates nucleotide substitutions per site. AMR, antimicrobial resistance; CR, community resident, a person with no known exposure to livestock; $\mathrm{IHO}$, industrial hog operation; MDRSA, multidrug resistant $S$. aureus; SSTI, skin and soft tissue infection. 
LA-SA CC9 isolates (0 SNP differences) at the same sampling time, which provides strong evidence of household transmission of LA-SA CC9 between IHO workers and their children. Second, clinical implications might arise regarding treatment regimens for LAMDRSA CC9 colonization and infection. Most (63.3\%; 31/49) LA-SA CC9 isolates from North Carolina were multidrug-resistant and carried multiple genes conferring resistance to antimicrobial drug classes critical for human medicine (23). Of note, the single LA-SA CC9 isolate from an IHO worker who reported a recent SSTI belonged to a putative transmission cluster, displayed an MDRSA phenotype, and previously was reported to display a high degree of pathogenicity compared with a hypervirulent community-associated MRSA strain, USA300 (GenBank accession no. CP000255), in a mouse model of SSTI (24).

Our results support potential transmission of LASA CC9 between IHO pigs and humans, and between humans and other humans, in the top 10 pig-producing counties in North Carolina. These findings are consistent with previous publications on LA-SA CC9 and other lineages of LA-SA. First, a separate analysis of LA-MRSA CC9 recovered from IHO pigs in China suggested potential transmission of LA-MRSA CC9 between pigs, humans, and cows (11). Second, an abundance of previous epidemiologic and WGS analyses support transmission of diverse lineages of LA-SA from pigs to humans, which can result in human SSTI and bloodstream infections $(8,10,25)$. Last, prior WGS analyses and epidemiologic studies have provided support for household transmission of LASA CC9 and CC398 between persons based on spatial, temporal, and genotypic overlap $(2,3,26)$. In our analysis, the exact transmission pathway remains unclear because we did not ascertain the direction of transmission or whether transmission occurred through direct or indirect contact.

Previous studies have suggested a $S$. aureus mutation rate of 5-10 SNPs per year per genome (27-30), but our threshold of 43 SNPs was justified for 2 reasons. First, our empirically derived SNP threshold was consistent with SNP-based thresholds used by others to identify suspected transmission of MRSA in clinical settings (31) and previous measures of withinperson S. aureus diversity (32). The robustness of our findings was supported when we used the median (32 SNPs), rather than maximum (43 SNPs), pairwise SNP distance among IHO pig cluster isolates as the SNP threshold for identification of putative transmission clusters. We excluded only 1 isolate from an IHO worker from putative transmission clusters, and the excluded isolate was not the SSTI-associated isolate (data not shown). Second, the aim of this study was to clarify whether any SNP-based evidence of transmission between IHO pig and human populations in North Carolina exists, rather than provide evidence of recent or incident transmission or to identify specific pathways of transmission. Using 43 SNPs as the threshold enabled us to observe potential direct or indirect transmission that might not be observed by using epidemiologic data alone. Investigations of $S$. aureus transmission conventionally combine epidemiologic and strain typing data, but these methods can fail to identify transmission links in cases in which spatial and temporal overlap is lacking (31). Using the epidemiologic data that were available to us, such as multiple $S$. aureus CC9 isolates from the same IHO, household, or individual, we observed SNP-based evidence of $S$. aureus CC9 clustering that would be expected biologically (Appendix Table 5).

Since 2016, tetracyclines have been the most heavily used antimicrobial drug class in the US pig production system, followed distantly by macrolides, lincosamides, aminoglycosides, streptogramins, and fluoroquinolones $(33,34)$. If antimicrobial-resistant CC9 strains were enriched through selective pressure, antimicrobial use in pig production possibly has played a role in the clonal expansion of LA-SA CC9 in North Carolina and other regions of the world. Of note, resistance to several of these antimicrobial drug classes was conferred by different AMR genes in C1, C2, and C3 (Figure 1; Appendix Table 4), highlighting different evolutionary pathways for adaptation to antimicrobial selection pressures in different regions of the world. Continued surveillance of IHO pigs and humans, including during and after regulatory and policy restrictions on antimicrobial use in animal agriculture, could provide critical insight into the potential contribution of antimicrobial use in the clonal expansion of LA-SA CC9 and its associated AMR genes in the United States.

The strengths of our study included using SNPbased analyses to examine the population structure and transmission dynamics of LA-SA CC9 among pigs and humans in a region of North Carolina with the highest density of IHOs in the United States (35), a region in which residents and IHO workers are actively expressing concerns about IHO-related exposures (36). Second, our study used SNP distance to classify human isolates closely related to IHO pig isolates, which is an improvement on previous studies that used spa-typing, MLST typing, absence of IEC genes (specifically scn), phenotypic AMR determination, or combinations of these techniques, to classify $S$. aureus isolates as livestock-associated $(2,3,12)$. 
Third, the use of a SNP-based definition for cluster analysis can capture the potential for transmission between animal and human populations that would have been missed by using more conventional epidemiologic methods alone (31).

Limitations of our study included that we were not able to provide evidence for directionality of transmission. We rooted our high-resolution phylogenetic tree at the midpoint; therefore, we are unsure if the most ancestral clade of S. aureus CC9 is of human or animal origin. In addition, whereas the SNP-based evidence for pig-to-human transmission could have been strengthened by spatial or temporal data linking pigs and workers at the same IHO, these data were not available because of efforts to protect the privacy of participants enrolled in the epidemiologic studies and because of limited access to IHOs in the United States (37). In contrast to countries in Europe, the lack of access to IHOs prevents us from assessing the generalizability of our results in the United States. We hypothesize that we would see even closer genetic relatedness between IHO worker and IHO pig LA-SO CC9 isolates collected from the same IHO at the same time. Last, our collection of $S$. aureus CC9 isolates was limited. The North Carolina collection was a convenience sample that identified S. aureus CC9 isolates from only 6 IHOs, which does not represent the full population of IHOs or pigs in North Carolina. Also, we excluded many isolates selected for WGS from SNP-analysis because they did not pass our quality control criteria (Appendix), potentially introducing bias into the studied isolate sample. Additional S. aureus CC9 isolates likely are available now in the NCBI Reference Sequence Database, but publicly available LA-SA CC9 sequence data were limited when we accessed the database for this study. A more representative dataset could provide more refined estimates on frequency of transmission in North Carolina and other regions of the world.

Despite these limitations, our results show a high degree of phylogenetic relatedness between IHO pig and human LA-SA CC9 isolates in the top 10 pig-producing counties in North Carolina. The presence of a highly pathogenic SSTI-associated LA-SA CC9 isolate with an MDRSA phenotype in a putative transmission cluster warrants future investigations into the disease burden associated with these strains in the United States. Future research could further improve or build on our findings by including environmental isolates and considering WGS analysis in conjunction with spatial and temporal data analysis to investigate the frequency of transmission, environmental exposure routes, and geographic extent of LA-SA CC9. Our reference dataset might be useful in future investigations of worker and community health concerns related to LA-SA CC9 dissemination and acquisition, both in North Carolina and in other regions of the United States with high densities of IHOs.

\section{Acknowledgments}

The authors thank Aaron Milstone for intellectual contributions to the manuscript drafts; the Rural Empowerment Association for Community Help (REACH) for their assistance with participant enrollment and collection of human nasal samples; and William Flowers, Sarah Blacklin, Asher Wright, Alexis Brown, Haley Keller, and Ralph A. Tripp for their assistance with collection of samples from pigs in North Carolina.

This study was funded by the Sherrilyn and Ken Fisher Center for Environmental Infectious Diseases Discovery Program at the Johns Hopkins University, School of Medicine, Department of Medicine, Division of Infectious Diseases (award no. 018HEA2013); a gift from the GRACE Communications Foundation; the National Institute for Occupational Safety and Health (NIOSH) pilot award from the Johns Hopkins NIOSH Education and Research Center (grant no. T42OH008428); and NIOSH grant no. K01OH010193 and National Science Foundation (NSF) grant no. 1316318 as part of the joint NSF-NIH-USDA Ecology and Evolution of Infectious Diseases program. P.R.R. was supported by NIOSH (grant no. T42OH008428) and a gift from the GRACE Communications Foundation. J.L., L.B.P., and M.A. received funding support from the National Institute of Allergy and Infectious Diseases (NIAID; grant no. R01AI101371). C.D.H. received funding from NIOSH (grant no. K01OH010193). E.W. received funding from " $\mathrm{Al}$ " Thrasher Award 10287; a gift from the GRACE Communications Foundation; the National Institute of Environmental Health Sciences (grant no. R01ES026973); NIAID (grant nos. R21AI139784, R43AI141265, and R01AI130066); and the National Science Foundation (grant no. 1316318), as part of the joint NSF-NIH-USDA Ecology and Evolution of Infectious Diseases program. M.L.N. received funding support as part of a Royster Society fellowship and a US Environmental Protection Agency Science to Achieve Results fellowship. M.F.D. received funding support from NIH (grant no. K01OD019918). L.S.M received funding support from NIH (grant nos. R01AR069502 and R01AR073665). D.C.L. received funding support through a gift from the GRACE Communications Foundation. The funders had no role in study design, data collection and analysis, decision to publish, or preparation of the manuscript. 
D.H. is the program manager and co-founder of the Rural Empowerment Association for Community Help (REACH), a 501(c)(3) not-for-profit organization located in Duplin County, North Carolina, and was a complainant in a Title VI administrative complaint against the North Carolina Department of Environmental Quality related to its statewide hog operation lagoon and spray field liquid waste management permitting system; D.H. has no potential personal financial gain from this administrative complaint, which is not directly related to the research described in this manuscript and is not a lawsuit or litigation.

L.S.M. is a full-time employee of Janssen Pharmaceuticals and may hold Johnson \& Johnson stock and stock options. He performed all work at his prior affiliation at Johns Hopkins University School of Medicine; has received prior grant support from AstraZeneca, Pfizer, Boehringer Ingelheim, Regeneron Pharmaceuticals, and Moderna Therapeutics; was a paid consultant for Armirall and Janssen Research and Development; was on the scientific advisory board of Integrated Biotherapeutics; and is a shareholder of Noveome Biotherapeutics, which are all developing therapeutics against infections, including S. aureus and other pathogens, and inflammatory conditions. All other authors declare that they have no actual or potential competing financial interests.

\section{About the Author}

Dr. Randad is a postdoctoral fellow in the department of Environmental Health and Engineering at Johns Hopkins University Bloomberg School of Public Health. His research focuses on infectious disease epidemiology and diagnostic development.

\section{References}

1. Rinsky JL, Nadimpalli M, Wing S, Hall D, Baron D, Price LB, et al. Livestock-associated methicillin and multidrug resistant Staphylococcus aureus is present among industrial, not antibiotic-free livestock operation workers in North Carolina. PLoS One. 2013;8:e67641. https:// doi.org/10.1371/ journal.pone.0067641

2. Hatcher SM, Rhodes SM, Stewart JR, Silbergeld E, Pisanic N, Larsen J, et al. The prevalence of antibiotic-resistant Staphylococcus aureus nasal carriage among industrial hog operation workers, community residents, and children living in their households: North Carolina, USA. Environ Health Perspect. 2017;125:560-9. https://doi.org/10.1289/EHP35

3. Nadimpalli ML, Stewart JR, Pierce E, Pisanic N, Love DC, Hall D, et al. Face mask use and persistence of livestockassociated Staphylococcus aureus nasal carriage among industrial hog operation workers and household contacts, USA. Environ Health Perspect. 2018;126:127005. https://doi.org/10.1289/EHP3453

4. Casey JA, Curriero FC, Cosgrove SE, Nachman KE, Schwartz BS. High-density livestock operations, crop field application of manure, and risk of community-associated methicillin-resistant Staphylococcus aureus infection in Pennsylvania. JAMA Intern Med. 2013;173:1980-90. https:// doi.org/10.1001/jamainternmed.2013.10408

5. Wardyn SE, Forshey BM, Farina SA, Kates AE, Nair R, Quick MK, et al. Swine farming is a risk factor for infection with and high prevalence of carriage of multidrug-resistant staphylococcus aureus. Clin Infect Dis. 2015;61:59-66. https://doi.org/10.1093/cid/civ234

6. Wardyn SE, Stegger M, Price LB, Smith TC. Whole-genome analysis of recurrent Staphylococcus aureus t571/ST398 infection in farmer, Iowa, USA. Emerg Infect Dis. 2018;24:153-4. https://doi.org/10.3201/eid2401.161184

7. Nadimpalli M, Stewart JR, Pierce E, Pisanic N, Love DC, Hall D, et al. Livestock-associated, antibiotic-resistant Staphylococcus aureus nasal carriage and recent skin and soft tissue infection among industrial hog operation workers. PLoS One. 2016;11:e0165713. https:/ / doi.org/ 10.1371/journal.pone.0165713

8. Larsen J, Petersen A, Larsen AR, Sieber RN, Stegger M, Koch A, et al.; Danish MRSA Study Group. Emergence of livestock-associated methicillin-resistant Staphylococcus aureus bloodstream infections in Denmark. Clin Infect Dis. 2017;65:1072-6. https://doi.org/10.1093/cid/cix504

9. Butaye P, Argudín MA, Smith TC. Livestock-associated MRSA and its current evolution. Curr Clin Microbiol Rep. 2016;3:19-31. https://doi.org/10.1007/s40588-016-0031-9

10. Chuang Y-Y, Huang Y-C. Livestock-associated methicillinresistant Staphylococcus aureus in Asia: an emerging issue? Int J Antimicrob Agents. 2015;45:334-40. https:/ / doi.org/ 10.1016/j.ijantimicag.2014.12.007

11. Zhou W, Li X, Osmundson T, Shi L, Ren J, Yan H. WGS analysis of ST9-MRSA-XII isolates from live pigs in China provides insights into transmission among porcine, human and bovine hosts. J Antimicrob Chemother. 2018;73:2652-61. https://doi.org/10.1093/jac/dky245

12. Davis MF, Pisanic N, Rhodes SM, Brown A, Keller H, Nadimpalli M, et al. Occurrence of Staphylococcus aureus in swine and swine workplace environments on industrial and antibiotic-free hog operations in North Carolina, USA: a One Health pilot study. Environ Res. 2018;163:88-96. https://doi.org/10.1016/j.envres.2017.12.010

13. Bankevich A, Nurk S, Antipov D, Gurevich AA, Dvorkin M, Kulikov AS, et al. SPAdes: a new genome assembly algorithm and its applications to single-cell sequencing. J Comput Biol. 2012;19:455-77. https:/ / doi.org/10.1089/ cmb.2012.0021

14. Enright MC, Day NPJ, Davies CE, Peacock SJ, Spratt BG. Multilocus sequence typing for characterization of methicillin-resistant and methicillin-susceptible clones of Staphylococcus aureus. J Clin Microbiol. 2000;38:1008-15. https://doi.org/10.1128/JCM.38.3.1008-1015.2000

15. Zankari E, Hasman H, Cosentino S, Vestergaard M, Rasmussen S, Lund O, et al. Identification of acquired antimicrobial resistance genes. J Antimicrob Chemother. 2012;67:2640-4. https://doi.org/10.1093/jac/dks261

16. Altschul SF, Gish W, Miller W, Myers EW, Lipman DJ. Basic local alignment search tool. J Mol Biol. 1990;215:403-10. https:/ / doi.org/10.1016/S0022-2836(05)80360-2

17. Sahl JW, Lemmer D, Travis J, Schupp JM, Gillece JD, Aziz M, et al. NASP: an accurate, rapid method for the identification of SNPs in WGS datasets that supports flexible input and output formats. Microb Genom. 2016;2:e000074. https:// doi.org/10.1099/mgen.0.000074

18. Croucher NJ, Page AJ, Connor TR, Delaney AJ, Keane JA, Bentley SD, et al. Rapid phylogenetic analysis of large samples of recombinant bacterial whole genome sequences 
using Gubbins. Nucleic Acids Res. 2015;43:e15-15. https://doi.org/10.1093/nar/gku1196

19. Guindon S, Dufayard J-F, Lefort V, Anisimova M, Hordijk W, Gascuel O. New algorithms and methods to estimate maximum-likelihood phylogenies: assessing the performance of PhyML 3.0. Syst Biol. 2010;59:307-21. https://doi.org/10.1093/sysbio/syq010

20. Guindon S, Gascuel O. A simple, fast, and accurate algorithm to estimate large phylogenies by maximum likelihood. Syst Biol. 2003;52:696-704. https:// doi.org/ 10.1080/10635150390235520

21. Tamura K, Peterson D, Peterson N, Stecher G, Nei M, Kumar S. MEGA5: molecular evolutionary genetics analysis using maximum likelihood, evolutionary distance, and maximum parsimony methods. Mol Biol Evol. 2011;28:27319. https://doi.org/10.1093/molbev/msr121

22. Magiorakos A-P, Srinivasan A, Carey RB, Carmeli $Y$, Falagas ME, Giske CG, et al. Multidrug-resistant, extensively drug-resistant and pandrug-resistant bacteria: an international expert proposal for interim standard definitions for acquired resistance. Clin Microbiol Infect. 2012;18:268-81. https://doi.org/10.1111/ j.1469-0691.2011.03570.x

23. World Health Organization. Critically important antimicrobials for human medicine, 6th revision. Geneva: The Organization; 2018 [cited 2020 Dec 18]. https:/ / www. who.int/foodsafety/publications/antimicrobials-sixth

24. Randad PR, Dillen CA, Ortines RV, Mohr D, Aziz M, Price LB, et al. Author correction: comparison of livestockassociated and community-associated Staphylococcus aureus pathogenicity in a mouse model of skin and soft tissue infection. Sci Rep. 2019;9:12811. https:// doi.org/10.1038/ s41598-019-46940-z

25. Price LB, Stegger M, Hasman H, Aziz M, Larsen J, Andersen PS, et al. Staphylococcus aureus CC398: host adaptation and emergence of methicillin resistance in livestock. mBio. 2012;3:e00305-11. https:/ / doi.org/10.1128/ mBio.00305-11

26. Bosch T, Verkade E, van Luit M, Landman F, Kluytmans J, Schouls LM. Transmission and persistence of livestockassociated methicillin-resistant Staphylococcus aureus among veterinarians and their household members. Appl Environ Microbiol. 2015;81:124-9. https://doi.org/10.1128/ AEM.02803-14

27. Harris SR, Feil EJ, Holden MTG, Quail MA, Nickerson EK, Chantratita N, et al. Evolution of MRSA during hospital transmission and intercontinental spread. Science. 2010; 327:469-74

28. Nübel U, Dordel J, Kurt K, Strommenger B, Westh H, Shukla SK, et al. A timescale for evolution, population expansion, and spatial spread of an emerging clone of methicillin-resistant Staphylococcus aureus. PLoS Pathog. 2010;6:e1000855. https:// doi.org/10.1371/ journal.ppat.1000855

29. Smyth DS, McDougal LK, Gran FW, Manoharan A, Enright MC, Song J-H, et al. Population structure of a hybrid clonal group of methicillin-resistant Staphylococcus aureus, ST239-MRSA-III. PLoS One. 2010;5:e8582. https://doi.org/ 10.1371/journal.pone.0008582

30. Young BC, Golubchik T, Batty EM, Fung R, Larner-Svensson $\mathrm{H}$, Votintseva AA, et al. Evolutionary dynamics of Staphylococcus aureus during progression from carriage to disease. Proc Natl Acad Sci U S A. 2012;109:4550-5. https://doi.org/10.1073/pnas.1113219109

31. Price JR, Golubchik T, Cole K, Wilson DJ, Crook DW, Thwaites GE, et al. Whole-genome sequencing shows that patient-to-patient transmission rarely accounts for acquisition of Staphylococcus aureus in an intensive care unit. Clin Infect Dis. 2014;58:609-18. https:/ / doi.org/10.1093/ $\mathrm{cid} / \mathrm{cit} 807$

32. Golubchik T, Batty EM, Miller RR, Farr H, Young BC, Larner-Svensson $\mathrm{H}$, et al. Within-host evolution of Staphylococcus aureus during asymptomatic carriage. PLoS One. 2013;8:e61319. https:// doi.org/10.1371/journal. pone.0061319

33. US Food and Drug Administration. 2016 summary report on antimicrobials sold or distributed for use in food-producing animals [cited 2020 Dec 18]. https://www.fda.gov/ media/109457/download

34. US Food and Drug Administration. 2017 summary report on antimicrobials sold or distributed for use in food-producing animals [cited 2020 Dec 18]. https:/ / www.fda.gov/ media/119332/download

35. US Department of Agriculture, North Carolina Department of Agriculture and Consumer Services. 2018 North Carolina agricultural statistics [cited 2020 Dec 18]. https:/ / quickstats. nass.usda.gov

36. Cole D, Todd L, Wing S. Concentrated swine feeding operations and public health: a review of occupational and community health effects. Environ Health Perspect. 2000;108:685-99. https://doi.org/10.1289/ehp.00108685

37. Ceryes CA, Heaney CD. "Ag-Gag" laws: evolution, resurgence, and public health implications. NEW Solut. 2019.28:664-82. https://doi.org/10.1177/1048291118808788

Address for correspondence: Pranay R. Randad, Department of Environmental Health and Engineering, Bloomberg School of Public Health, Johns Hopkins University, 615 N Wolf St, Baltimore, MD 21205, USA; email: prandad1@jhmi.edu 